\title{
Optical Fiber Sensor Based on Second-Order Superstructure Fiber Bragg Grating
}

\author{
Hung-Ying Chang, ${ }^{*}$ Ming-Yue Fu, ${ }^{1}$ and Wen-Fung Liu ${ }^{2}$ \\ Ph.D. Program in Electrical and Communications Engineering, Feng-Chia University, \\ No. 100, Wenhwa Rd., Seatwen, Taichung, Taiwan, R.O.C. \\ ${ }^{1}$ Department of Avionics Engineering, Air Force Academy, \\ No. 1, Jieshou W. Rd., Gangshan Dist., Kaohsiung, Taiwan, R.O.C. \\ ${ }^{2}$ Department of Electrical Engineering, Feng-Chia University, \\ No. 100, Wenhwa Rd., Seatwen, Taichung, Taiwan 40724, R.O.C. \\ (Received August 31, 2015; accepted February 3, 2016)
}

Keywords: fiber sensor, fiber Bragg grating, long-period grating, superstructure fiber Bragg grating

An optical fiber sensor based on a second-order superstructure fiber Bragg grating (SFBG) is experimentally investigated. In general, a first-order SFBG has been available for many years and has been widely applied in optical fiber communication systems and fiber sensing systems. An SFBG is composed of a fiber Bragg grating (FBG) and a long-period grating (LPG), so it can be regarded as a periodically modulated FBG. In this study, to the best of our knowledge, a second-order SFBG is successfully fabricated for the first time. Moreover, we are the first to experimentally demonstrate that the refractive index sensitivities of a second-order SFBG sensor are larger than those of the first-order SFBG. This configuration and technique can provide simplestructure, low-cost, and high-sensitivity innovative devices for application to refractive index measurement systems.

\section{Introduction}

Since the first fabrication of a fiber grating by transverse holographic technology in $1989,{ }^{(1)}$ the fiber grating has been developed for various applications such as telecommunications and fiber sensor systems. ${ }^{(2-4)}$ Optical fiber sensors are currently attracting significant interest for civil engineering applications and biosensors. The sensors have the potential to be used to measure pressure and deformations in numerous environments, such as smart structures or composite materials in bridges or dams. ${ }^{(5,6)}$ For these applications, different types of fiber gratings can be designed and fabricated according to the index modulation distribution and grating period design. ${ }^{(2)}$ In general, a single channel fiber grating is more often used for sensing only one physical parameter by measuring the Bragg wavelength shift. For fiber sensing measurement, ${ }^{(3-5)}$ the variation of light signal intensity in the sensing head can be used to detect changes in external physical or chemical parameters. For a multichannel grating, a superstructure fiber Bragg grating (SFBG) of multireflection channels has been used to simultaneously measure different physical parameters. An SFBG is composed of a Bragg grating and a long-period grating, and it can be regarded as a periodically modulated fiber Bragg grating (FBG). Thus, counterdirectional guided mode coupling

*Corresponding author: e-mail: hungying.chang@gmail.com 
is introduced in regularly separated wavelength channels, resulting in a comblike reflection spectrum. ${ }^{(3)}$ On the basis of the codirectionality and the counterdirectional coupling of an SFBG, a fiber sensor with a single sensing component has been used to measure multiple parameters simultaneously, such as axial strain, pressure, temperature, and curvature. ${ }^{(4-6)}$

In this study, a second-order SFBG was successfully fabricated for the first time. Then, we experimentally demonstrated that the refractive index (RI) sensitivity of this sensor is larger than that of the first-order SFBG.

\section{Second-Order SFBG Fabrication}

According to the grating period, fiber gratings can be divided into short-period gratings (or FBGs) and long-period gratings (LPGs). In general, the period $\Lambda_{\mathrm{FBG}}$ of an FBG is about $1 \mu \mathrm{m}$, and the range of an LPG pitch is between several tens of to several hundred micrometers. FBGs are based on the grating phase-matching condition to create a forward-propagating core mode that can be coupled into a reverse-propagating core mode. The Bragg wavelength $\lambda_{\mathrm{B}}\left(=2 n_{\mathrm{co}} \Lambda_{\mathrm{FBG}}\right)$ can be derived from the grating period $\Lambda_{\mathrm{FBG}}$ and the refractive index of the core mode, $n_{\mathrm{co}}$. LPGs exist only for codirectional coupling owing to the smaller grating wave vector. The resonant wavelengths $\lambda_{\text {LPG }}$ depend on the difference between the refractive indices of the core mode, $n_{\mathrm{co}}$, and the nth cladding mode, $n_{\mathrm{cl}(n)}$, as well as the grating period $\Lambda_{\mathrm{LBG}}$. The relationship can be expressed as $\lambda_{\mathrm{LPG}}=\left(2 n_{\mathrm{co}}-\right.$ $\left.n_{\mathrm{cl}(n)}\right) \Lambda_{\mathrm{LBG}}$.

When an FBG is fabricated under a large modulated index change by continuous UV exposure, the reflecting grating becomes saturated and the spectrum broadens because the incident wave is completely reflected and reaches the end of the grating. The strongly saturated grating is no longer sinusoidal, and the peak index regions are flattened, whereas the valleys in the perturbation index distribution are sharpened. As a result, a second-order Bragg reflection wavelength is observed at about one-half the value of the fundamental Bragg wavelength and at other shorter wavelengths for high-order modes. ${ }^{(8)}$ Figure 1 shows a second-order FBG wavelength at $1533 \mathrm{~nm}$ and another reflective wavelength at $1532.5 \mathrm{~nm}$ for a high-order mode.

An SFBG comprises a fiber Bragg grating and a long-period grating as a special kind of fiber grating fabricated by the periodically modulated exposure of the FBG, as shown in Fig. 2.

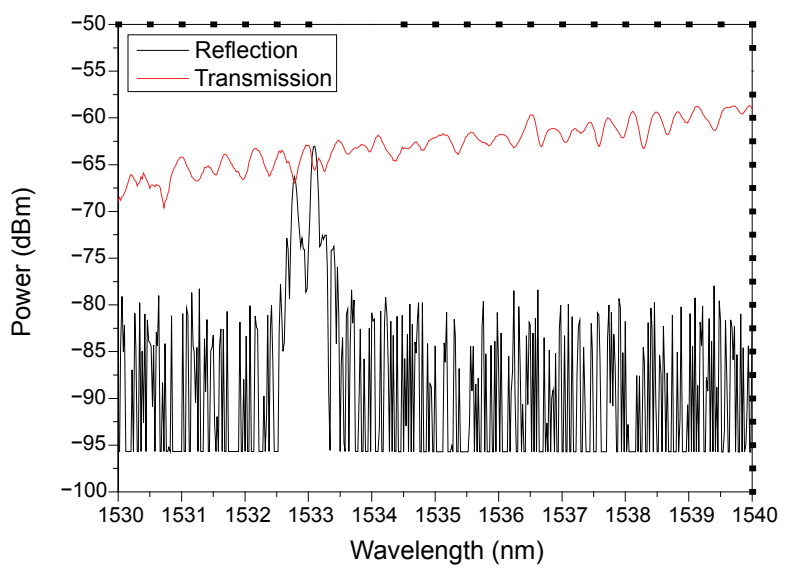

Fig. 1. (Color online) Optical spectrum of a secondorder FBG.

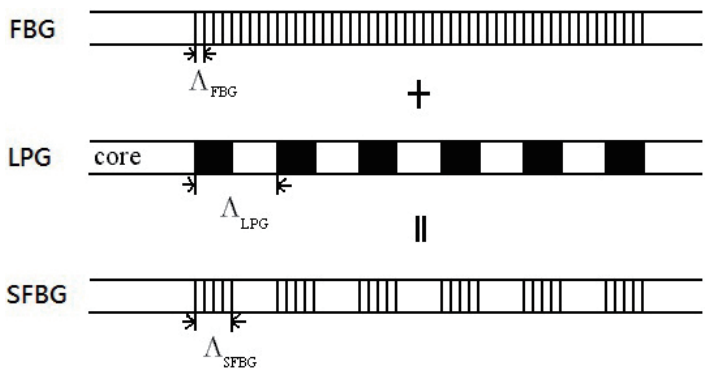

Fig. 2. Structure of SFBG. 
By using a Fourier transform, the spatial frequency contents of the superstructure can be approximated by a comb of delta functions centered on the Bragg frequency. Based on the coupled mode theory, each Fourier component contributes a reflectivity peak to the reflection spectrum of an SFBG. Its channel spacing and reflectivity depend on the period grating and index intensity of the fiber Bragg grating, respectively. Figure 3 shows the reflective spectrum of a first-order SFBG. By the same method as for the fabrication of a second-order FBG, a second-order SFBG is fabricated under a large modulated index change by UV exposure. Every second-order reflective wavelength is neighbored by a shorter reflective wavelength for high-order modes as shown Fig. 4 . When the refractive index surrounding the grating is changed, the reflective wavelength is changed due to the RI change of the cladding mode. In this way, the RI can be detected based on the gratings.

\section{Experimental Setup and Results}

The FBG used for this study was fabricated on a hydrogen-loaded single-mode fiber-type PS1500 from Fiber-Core Corporation using a writing technology based on the superposition of a phase mask (1.071 $\mu \mathrm{m}$ for first order, $2.0611 \mu \mathrm{m}$ for second order) illuminated with a $\mathrm{KrF}$ excimer laser at $248 \mathrm{~nm}$. For the SFBG, the writing setup involved placing the amplitude mask $(400 \mu \mathrm{m})$ in front of the phase mask. The length of a grating was varied by adjusting the gap in the slit located in front of the amplitude mask. The spectral measurements were performed using an amplified spontaneous emission (ASE) source over a range from 1500 to $1600 \mathrm{~nm}$, and an optical spectrum analyzer (OSA). The first-order SFBG and second-order SFBG refractive spectra are shown in Figs. 3 and 4, respectively.

To prevent temperature cross-sensitivity, the experiment temperature was controlled to $28{ }^{\circ} \mathrm{C}$. The reflective wavelength shift was investigated using various RI oils to surround the cladding region to monitor the variation in the grating spectrum. When the RI of the oil increases from 1.30 to 1.38 for a first-order SFBG, the reflective wavelength shift can be observed using an OSA. In this experiment, we selected the wavelength of $1536.4 \mathrm{~nm}$ to demonstrate the sensing scheme. The experimental results for the different refractive indices at 1.3, 1.32, 1.34, 1.36, and 1.38 are shown

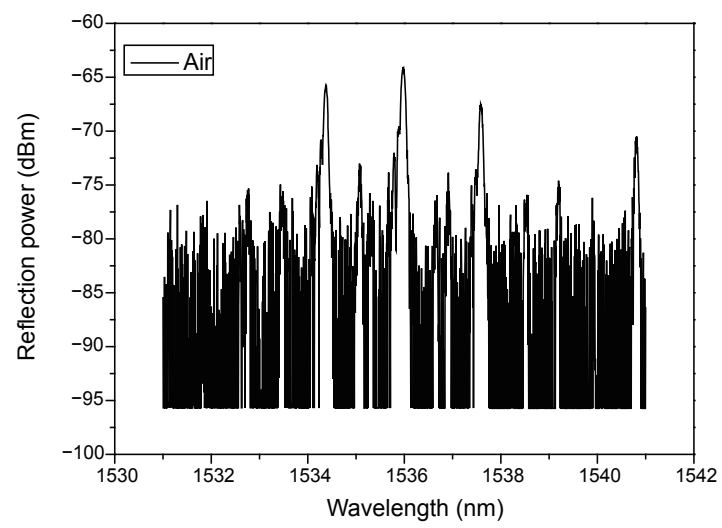

Fig. 3. Optical spectrum of a first-order SFBG.

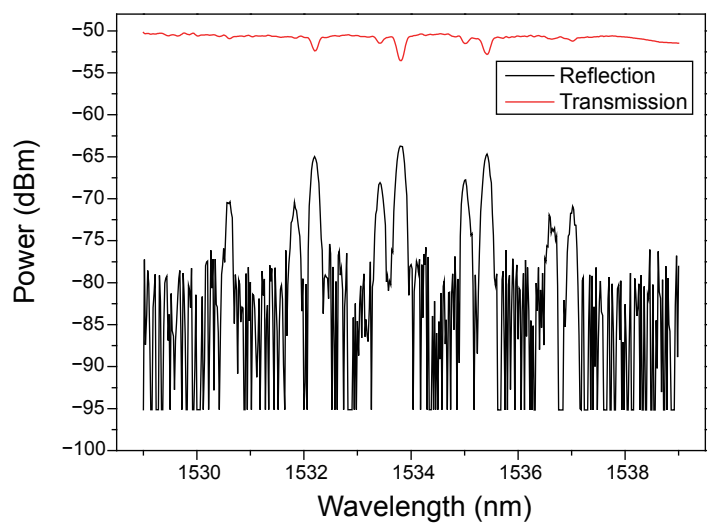

Fig. 4. (Color online) Optical spectrum of a secondorder SFBG. 
in Fig. 5. From Fig. 5, we can clearly observe that the wavelength peaks shift toward the larger wavelength side as the refractive index increases. This phenomenon is due to the effective cladding index being increased by the increase in the index surrounding the fiber grating. Figure 6 indicates the relationship of the peak wavelengths of a first-order SFBG versus the range of variation in the index. The RI sensitivities are $0.125 \mathrm{~nm} /$ unit index.

For a second-order SFBG within a refractive index variation ranging from 1.30 to 1.38 , the wavelengh peak shifted from 1532.846 to $1532.894 \mathrm{~nm}$, as shown Fig. 7. The peak wavelength shift is proportional to the RI, as shown Fig. 8, which corresonds to a sensitivity of $0.605 \mathrm{~nm} / \mathrm{unit}$ index. From these experimental results, we demonstrated that the RI sensitivity of a second-order SFBG is larger than that of a first-order SFBG.

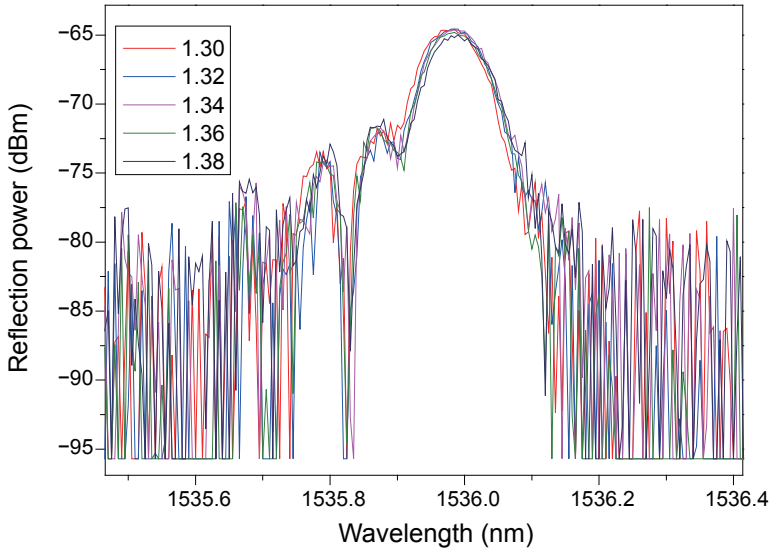

Fig. 5. Reflection spectra for measuring the different refractive indices surrounding a first-order SFBG.

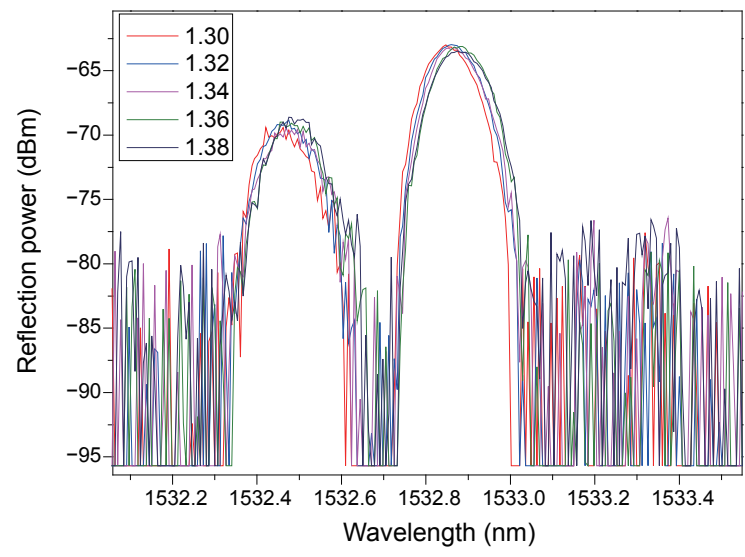

Fig. 7. Reflection spectra for measuring the different refractive indices surrounding a secondorder SFBG.

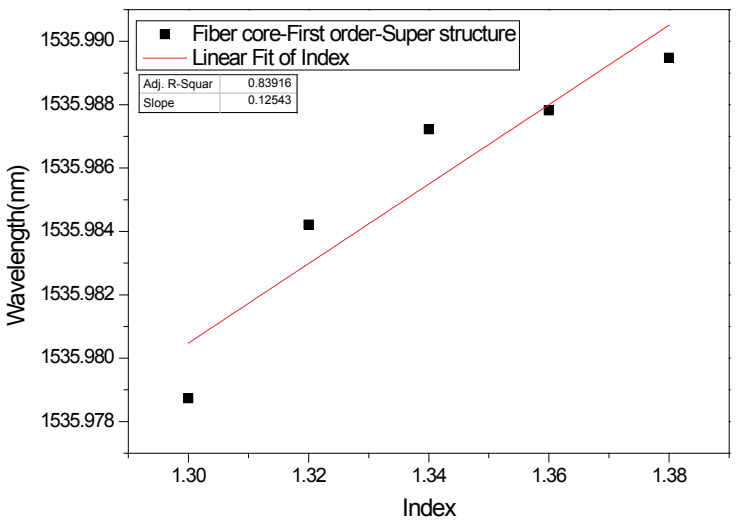

Fig. 6. (Color online) Relationship between the refractive index and the reflective wavelength shift of a first-order SFBG.

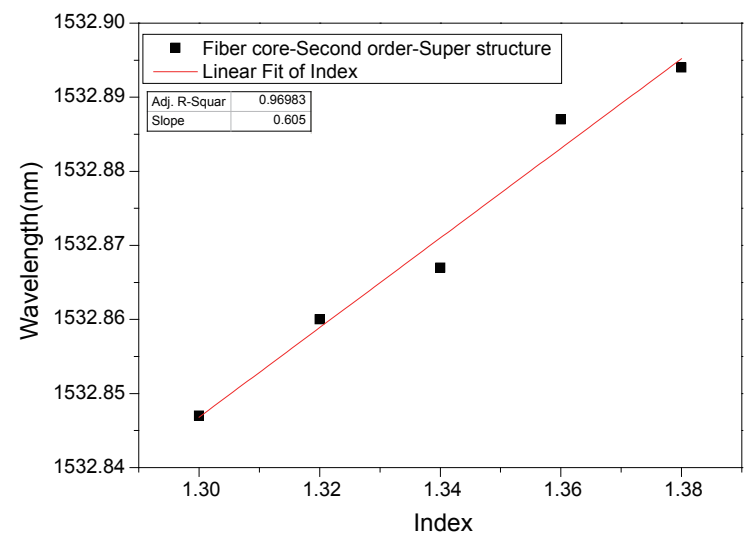

Fig. 8. (Color online) Relationship between the refractive index and the reflective wavelength shift of a second-order SFBG. 


\section{Conclusions}

In this study, we have successfully fabricated for the first time a second-order SFBG. Moreover, we demonstrated that the RI sensitivity of a second-order SFBG is larger that of a first-order SFBG. The sensitivity of a second-order SFBG is about 5 times that of a first-order SFBG. The sensitivity is improved by increasing the order of the fiber gratings.

\section{Acknowledgements}

The authors would like to specifically thank the Ministry of Science and Technology, Taiwan, for sponsoring this research under Contract Nos. MOST 103-2221-E-035-006-MY3 and 103-2221E-013-001.

\section{References}

1 G. Meltz, W. W. Morey, and W. H. Glen: Opt. Lett. 14 (1989) 823.

2 B. O. Guan, H. Y. Tam, X. M. Tao, and X. Y. Dong: IEEE Photon. Technol. Lett. 12 (2000) 675.

3 M. G. Xu, L. Reekie, Y. T. Chow, and J. P. Dakin: Electron. Lett. 29 (1993) 398.

4 C. Fernandez-Valdivielso, I. R. Matias, and F. J. Arregui: Elsevier Sci., A 101 (2002) 107.

5 B. O. Guan, H. Y. Tam, S. L. Ho, W. H. Chung, and X. Y. Dong: Electron. Lett. 36 (2000) 1018.

6 H. Chi, X. M. Tao, and D. X. Yang: Opt. Lett. 26 (2001) 1949.

7 H. J. Patrick, G. M. Williams, A. D. Kersey, J. R. Pedrazzani, and A. M. Vengsarkar: IEEE Photon. Technol. Lett. 8 (1996) 1223.

8 K. O. Hill: J. Lightwave Technol. 15 (1997) 1263.

9 A. Iadicicco, A. Cusano, A. Cutolo, R. Bernini, and M. Giordano: IEEE Photon. Technol. Lett. 16 (2004) 1149.

10 J. F. Ding, A. Ping Zhang, L. Y. Shao, J. H. Yan, and S. He: IEEE Photon. Technol. Lett. 17 (2005) 1247.

11 H. J. Patrick, A. D. Kersey, and F. Bucholtz: J. Lightwave Technol. 16 (1998) 1606. 